\title{
Taking some heat off the NDCs? The limited potential of additional short-lived climate forcers' mitigation
}

\author{
Mathijs Harmsen ${ }^{1,2}$ (D) Oliver Fricko $^{3}$ - Jérôme Hilaire ${ }^{4,5}$. Detlef $P$. van Vuuren ${ }^{1,2}$. \\ Laurent Drouet $^{6}$ - Olivier Durand-Lasserve ${ }^{7}$. Shinichiro Fujimori ${ }^{8,9}$. \\ Kimon Keramidas ${ }^{10} \cdot$ Zbigniew Klimont $^{3}$. Gunnar Luderer ${ }^{4}$. Lara Aleluia Reis ${ }^{6}$. \\ Keywan Riahi ${ }^{3} \cdot$ Fuminori Sano ${ }^{11}$ - Steven J. Smith ${ }^{12}$
}

Received: 10 October 2017 / Accepted: 10 April 2019 /Published online: 17 June 2019

(C) The Author(s) 2019

\begin{abstract}
Several studies have shown that the greenhouse gas reduction resulting from the current nationally determined contributions (NDCs) will not be enough to meet the overall targets of the Paris Climate Agreement. It has been suggested that more ambition mitigations of short-lived climate forcer (SLCF) emissions could potentially be a way to reduce the risk of overshooting the 1.5 or $2{ }^{\circ} \mathrm{C}$ target in a cost-effective way. In this study, we employ eight state-of-the-art integrated assessment models (IAMs) to examine the global temperature effects of ambitious reductions of methane, black and organic carbon, and hydrofluorocarbon emissions. The SLCFs measures considered are found to add significantly to the effect of the NDCs on short-term global mean temperature (GMT) (in the year 2040: -0.03 to $-0.15^{\circ} \mathrm{C}$ ) and on reducing the short-term rate-of-change (by -2 to $15 \%$ ), but only a small effect on reducing the maximum temperature change before 2100 . This, because later in the century under assumed ambitious climate policy, SLCF mitigation is maximized, either directly or indirectly due to changes in the energy system. All three SLCF groups can contribute to achieving GMT changes.
\end{abstract}

\section{Introduction}

In the 2015 Paris Agreement on Climate Change, parties have agreed to limit the global mean temperature (GMT) rise to well below $2{ }^{\circ} \mathrm{C}$ relative to pre-industrial levels and to pursue efforts to further limit this increase to a maximum of $1.5{ }^{\circ} \mathrm{C}$ (UNFCCC 2015b).

This article is part of a Special Issue reporting results from the "Energy Modeling Forum (EMF)-30 Study on Short-Lived Climate Forcers (SLCF) and Air Quality” edited by John Weyant, Steven J Smith, and Zbigniew Klimont

Electronic supplementary material The online version of this article (https://doi.org/10.1007/s10584-01902436-3) contains supplementary material, which is available to authorized users.

Mathijs Harmsen

j.h.m.harmsen@uu.nl

Extended author information available on the last page of the article 
Countries around the world have currently proposed emission reductions towards this goal, termed nationally determined contributions (NDCs) (UNFCCC 2015a). However, studies show that ambitions in the NDCs are too weak to reach greenhouse gas (GHG) reduction levels consistent with a 1.5 or $2{ }^{\circ} \mathrm{C}$ target (Den Elzen et al. 2016; Fawcett et al. 2016; Rogelj et al. 2016; UNEP 2016a; Vandyck et al. 2016). This means that the ambition of the NDCs needs to be strengthened to reach the goals of the Paris Agreement. One option would be to specifically strengthen non- $\mathrm{CO}_{2}$-related action with respect to short-lived climate forcers (SLCFs), of which the most relevant are methane $\left(\mathrm{CH}_{4}\right)$, ozone $\left(\mathrm{O}_{3}\right)$, black carbon (BC), and hydrofluorocarbons (HFCs).

This strategy of reducing SLCFs has been suggested earlier as a low-cost and effective mitigation policy option in the absence of large scale $\mathrm{CO}_{2}$ mitigation in the short term (Shindell et al. 2012; UNEP and WMO 2011; Victor et al. 2012). Several studies also indicated there is merit in reducing SLCFs as long as those reductions are additional to those of long-lived climate forcers (Shindell et al. 2012, 2017; Shoemaker et al. 2013; Smith and Mizrahi 2013; UNEP and WMO 2011). SLCF mitigation could in such case reduce short-term climate impacts (Bowerman et al. 2013).

The positive evaluation of SLCF strategies has also been criticized. First, SLCF reductions could potentially divert attention from $\mathrm{CO}_{2}$ mitigation, which is essential for mitigating temperature increase in the long term (Pierrehumbert 2014; Rogelj et al. 2014; Shoemaker et al. 2013). Moreover, the originally estimated impact of SLCF policy on global temperature change (Shindell et al. 2012) was found to be relatively modest in subsequent studies (Smith and Mizrahi 2013; Stohl et al. 2015; Strefler et al. 2014). Partly, this is based on higher estimated emission reductions of SLCFs in mitigation scenarios, resulting from the co-mitigation of $\mathrm{CO}_{2}$ and SLCFs, particularly of $\mathrm{CH}_{4}$. This lowers the added benefit of SLCF policy. In addition, it was shown that the co-mitigation of $\mathrm{BC}$ and climate cooling agents (e.g. sulphur dioxide $\left(\mathrm{SO}_{2}\right)$ and organic carbon (OC)) resulting from SLCF policies can dramatically reduce the effectiveness of these policies (Rogelj et al. 2014).

This study provides the first multi-model assessment of the potential contribution of intensified SLCF policy to the NDCs, in terms of limiting global temperature change. Eight state-of-the-art integrated assessment models (IAMs) have been employed to analyse NDC scenarios with and without strong additional SLCF policy, for both an NDC extrapolated and strong climate policy case, limiting the GMT to an increase of $2{ }^{\circ} \mathrm{C}$ in 2100 . This model comparison provides an assessment of potential regional and sectoral emission reductions and the resulting global implications on the short-term climate. In addition, the NDCs and national plans of the G20 countries have been assessed in terms of effectiveness of SLCF emission controls and compared with the model projections.

\section{Methods}

This study has been performed as part of the 30th Energy Modelling Forum (EMF30), an international collaboration project that aims to understand the potential role of SLCF mitigation in climate policy (EMF 2017). The EMF30 studies are based on an IAM scenario comparison, with a consistent experimental setup across multiple models. This paper also builds on that scenario comparison. 


\subsection{Scenario analysis}

Seven scenarios from EMF30 are relevant for this study's NDC analysis (see Table 1).

The first set of scenarios describes the situation without additional SLCF reduction. The "reference" scenario represents a no climate policy baseline scenario with default model improvements in methane emission factors and pollutant emission controls consistent with the current air quality policy extended to 2050 and model-specific baseline evolution beyond. The "NDC" scenario forms a variant of this scenario in which the NDCs are implemented until 2030 (currently the final target year). Beyond 2030, this scenario assumes a constant climate policy ambition. Note that this scenario already includes SLCF mitigation as SLCF GHGs $\left(\mathrm{CH}_{4}\right.$ and $\left.\mathrm{HFCs}\right)$ are reduced under a $\mathrm{CO}_{2}$-equivalent $\left(\mathrm{CO}_{2}\right.$-eq. $)$ price. The "NDC $+2 \mathrm{degC}$ " follows the NDC scenario until 2030, with strong mitigation thereafter, leading to a maximum temperature increase of $2{ }^{\circ} \mathrm{C}$ in 2100 . In this scenario, after 2030, the SLCF GHGs are increasingly reduced under a rising $\mathrm{CO}_{2}$-eq. price.

The second set of scenarios specifically looks into the impact of SLCFs. The "NDC+ SLCF" scenario forms a variant of the NDC scenario, in which additional, strong SLCF mitigation is introduced, ramped up towards 2030, and then equally ambitious thereafter (see Table 1 for detailed description). Note that with "equal ambition," emissions can further decrease towards 2100, as the maximum feasible reduction of SLCFs is time-dependent and expected to increase due to technological progress and removal of implementation barriers. The "NDC $+2 \operatorname{deg} C+\mathrm{SLCF}$ " scenario is equal to the $\mathrm{NDC}+2 \mathrm{degC}$ scenario (i.e. with the same carbon budget and price), but with additional, strong SLCF mitigation, ramped up towards 2030 and then equally ambitious thereafter. The "NDC $+2 \operatorname{deg} \mathrm{C}+\mathrm{SLCF}+\mathrm{HFCs}$ " is similar to NDC $+2 \operatorname{degC}+\mathrm{SLCF}$, but with additional HFC reductions.

Finally, the "Frozen-EF" is a diagnostic scenario with constant SLCF emission factors from 2010 onward, assuming no emissions control advancements beyond 2010. The scenario is used to diagnose the degree of emission control in the reference scenario.

Eight IAM models have taken part in the NDC analysis (see Supplement S1 for model descriptions): AIM/CGE, DNE21+, ENV-Linkages, IMAGE, MESSAGE-GLOBIOM, POLES, REMIND, and WITCH-GLOBIOM. All models have been widely used in climate policy research (i.e. Kriegler et al. 2014; Riahi et al. 2017; Tavoni et al. 2014) and comprise comprehensive global representations of the human economic system and associated emissions. They represent a broad spectrum of modelling approaches and model assumptions, such as the number of world regions, the level of foresight in determining optimal climate policy strategies, technological detail in the energy and land-use sectors, the representation of mitigation options, and substitutability of energy carriers.

In the baseline (reference), all models used the so-called SSP2 scenario (Riahi et al. 2017), a medium development scenario. To ensure consistency in the climate related results, the climate parameters have been assessed using the same climate model for all the models and scenarios: MAGICC6 (Meinshausen et al. 2011). In terms of its non- $\mathrm{CO}_{2}$ forcer representation, this simple climate model projects results within the range of those of the complex climate models it emulates (Harmsen et al. 2015) and is thus considered suitable for this exercise.

The model comparison provides an assessment of regional and sectoral implications for emissions of SLCFs. The mitigation measures apply to the SLCFs with the largest potential climate impact: $\mathrm{CH}_{4}, \mathrm{BC}$, and $\mathrm{HFCs}$. For $\mathrm{CH}_{4}$ and $\mathrm{HFCs}$, all mitigation measures represented by the models are included. For $\mathrm{BC}$, the focus is on the residential and transport sectors, where 


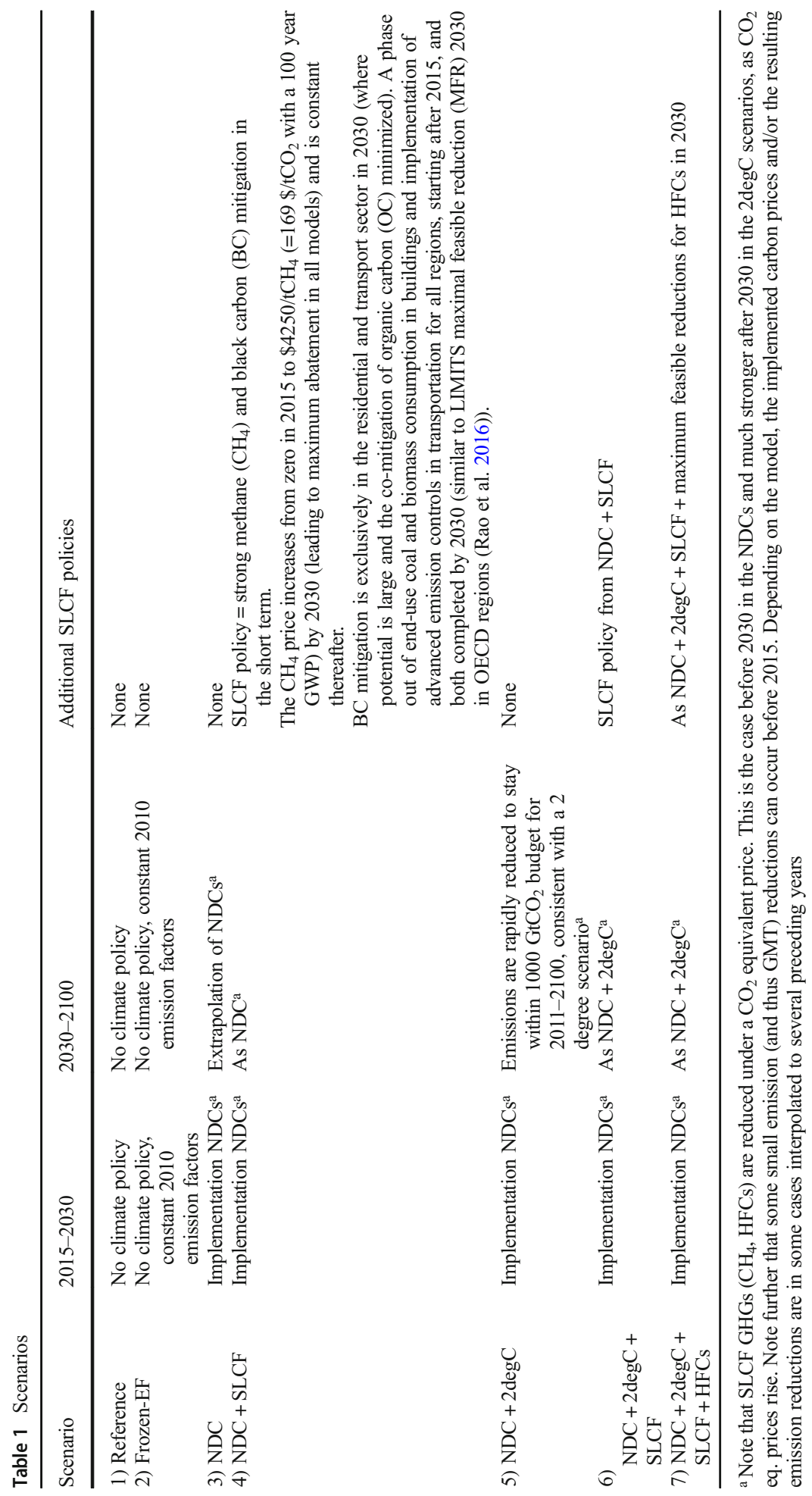


potential is large and the co-mitigation of OC, a climate cooling agent, is minimized (Bond et al. 2013).

The following indicators have been used to assess the climate impact of additional SLCF policy:

- GMT in 2040. This indicator shows the effect of SLCF policy in the near-term, where the GMT difference between SLCF policy and no-policy cases is generally large. The indicator is relevant for both the NDC extrapolated as NDC $+2 \mathrm{degC}$ case, considering short-term climate impacts and adaptation efforts.

- Peak GMT before 2100. This parameter is only considered relevant in the NDC $+2 \mathrm{degC}$ case, where there is a maximum temperature before 2100 , which can potentially be lowered by SLCF policy (while in the NDC extrapolated case the temperature is constantly increasing in all scenarios)

- Peak GMT rate of change before 2100. This parameter is potentially relevant in both the $\mathrm{NDC}$ extrapolated as in the NDC $+2 \mathrm{degC}$ case, where the highest rates of changes are expected to occur in the next two decades and can thus potentially be lowered by SLCF policy.

For the last two indicators, the peak years have been determined in all scenarios.

In addition, the contribution of the individual forcers to the potential cooling effect of SLCF policy has been assessed, by comparing the situations with and without additional SCLF policy in terms of radiative forcing (RF) per specific SLCF.

\section{Results}

\subsection{Emission pathways}

Figure 1 gives a general overview of the global emission pathways in the NDC scenarios, compared with the no-climate policy reference and frozen emission factor cases (model specific results are provided in Supplement S4; sectoral and regional emissions are further discussed in the next section).

By design, $\mathrm{CO}_{2}$ emissions are the same in the no-SLCF and SLCF policy cases. Any feedbacks from SLCF policy on $\mathrm{CO}_{2}$ emissions have been neglected. For all models, $\mathrm{CO}_{2}$ emissions follow one of three distinct pathways, increasing emissions in the reference case, stable to declining emissions in the NDC scenarios, and strongly declining emissions after 2030 in the $\mathrm{NDC}+2 \operatorname{deg} \mathrm{C}$ case. $\mathrm{CO}_{2}$ reduction measures can indirectly reduce $\mathrm{CH}_{4}$ and to a much lesser extent $\mathrm{BC}$ emissions, via the reduction of fossil fuel production and use and the reduction of deforestation (Rogelj et al. 2014). This $\mathrm{CO}_{2}$ indirect effect reduces the SLCF reduction potential in the mitigation scenarios.

All models project that without climate policy (reference in Fig. 1), $\mathrm{CH}_{4}$ emissions in 2030 are higher than in 2010. In the NDC scenario, 2030 emissions are comparable to those in 2010, although model results differ. Only limited (non-climate policy related) reductions in $\mathrm{CH}_{4}$ emission factors are assumed in the reference case, indicated by the relatively small difference with the frozen emission factor scenario. The NDCs are projected to lead to a decrease in emissions compared with the reference case, even in the absence of additional SLCF policy (see next section for a detailed description). 

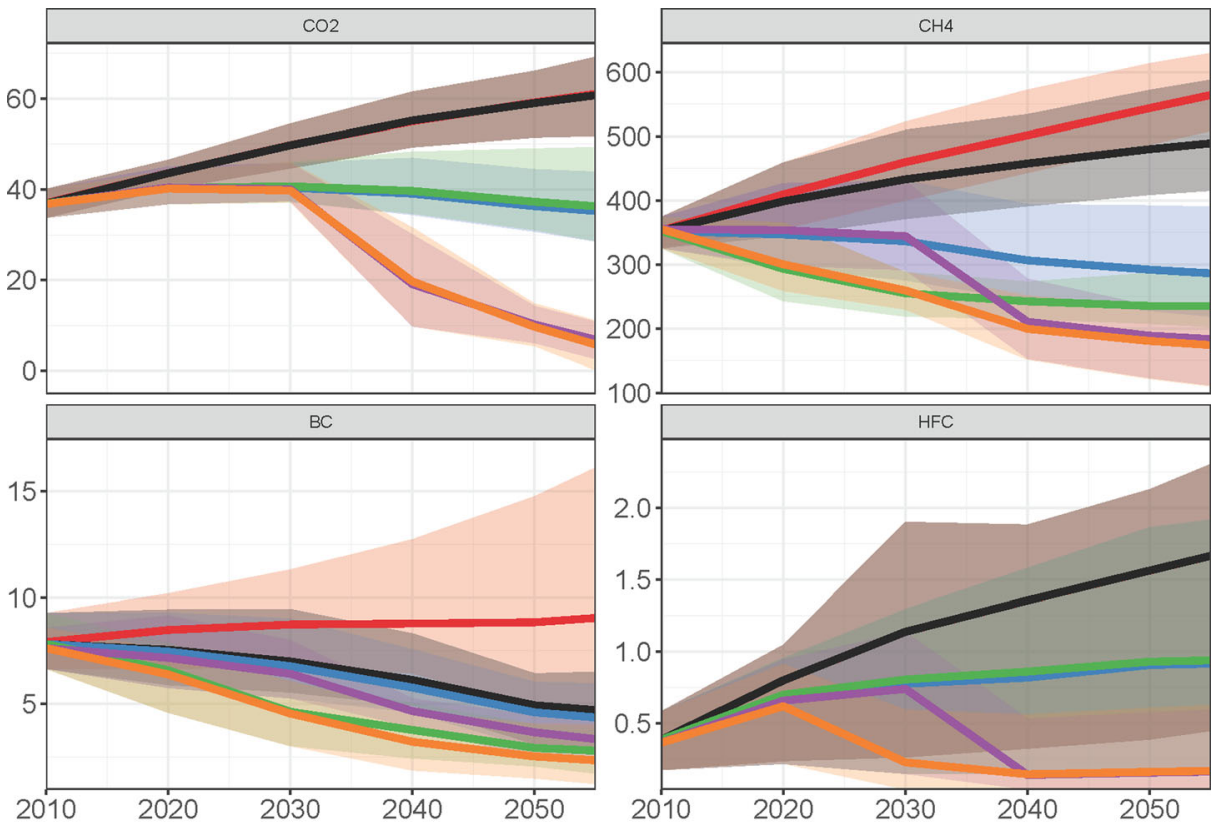

scenario

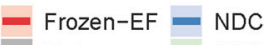

Reference $=\mathrm{NDC}+\mathrm{SLCF}=\mathrm{NDC}+2 \mathrm{deg}+\mathrm{SLCF}+\mathrm{HFC}$

Fig. 1 Global emission pathways in the SLCF scenarios, for $\mathrm{CO}_{2}$ and the three emitted SLCF groups, shown till the year 2055 (units: $\mathrm{Gt} \mathrm{CO}_{2}, \mathrm{Mt} \mathrm{CH}_{4}, \mathrm{Mt} \mathrm{BC}$, Mt HFC134a eq.). "NDC + degC + SLCF" is not shown, because it follows the same pattern as "NDC $+\operatorname{degC}+\mathrm{SLCF}+\mathrm{HFC}$ " for $\mathrm{CO}_{2}, \mathrm{CH}_{4}$, and $\mathrm{BC}$. Lines represent the model mean, areas the full model range (see Supplement $\mathrm{S} 4$ for the model specific emission pathways)

All models consistently project steadily decreasing BC emissions for the next decades in all scenarios. Even in a no-climate policy reference scenarios as SSP2, a considerable decrease in emission factors is expected (by $23 \%$ in 2030, model mean) resulting from stricter air pollutants emission legislation and increasing access to clean energy for cooking in growing economies (Rao et al. 2017). This also reduces the mitigation potential in 2030 in the NDC scenarios. The projected difference in emissions between the reference and NDC scenario is small $(<5 \%)$, since $\mathrm{BC}$ is not included as an offset under the Kyoto Protocol and mitigation efforts concentrate on Kyoto GHGs in the NDC case. In the NDC scenario, the very limited $\mathrm{CO}_{2}$ reduction measures also do not have much impact on $\mathrm{BC}$.

The projected $\mathrm{HFC}$ emissions differ from $\mathrm{CH}_{4}$ and $\mathrm{BC}$ in the sense that they are not indirectly mitigated by $\mathrm{CO}_{2}$ reduction measures, but only directly, depending on the climate policy stringency (i.e. the carbon price level). In the next section, the model specific assumptions and results are discussed.

\subsubsection{Methane emissions}

Figure 2 shows the sectoral and regional $\mathrm{CH}_{4}$ emissions for the NDC and reference scenarios. In the bar charts, the emissions and emission reductions are subdivided into 9 sectors and 5 aggregated world regions given for 2030 . Here, no distinction is made between the two degree 

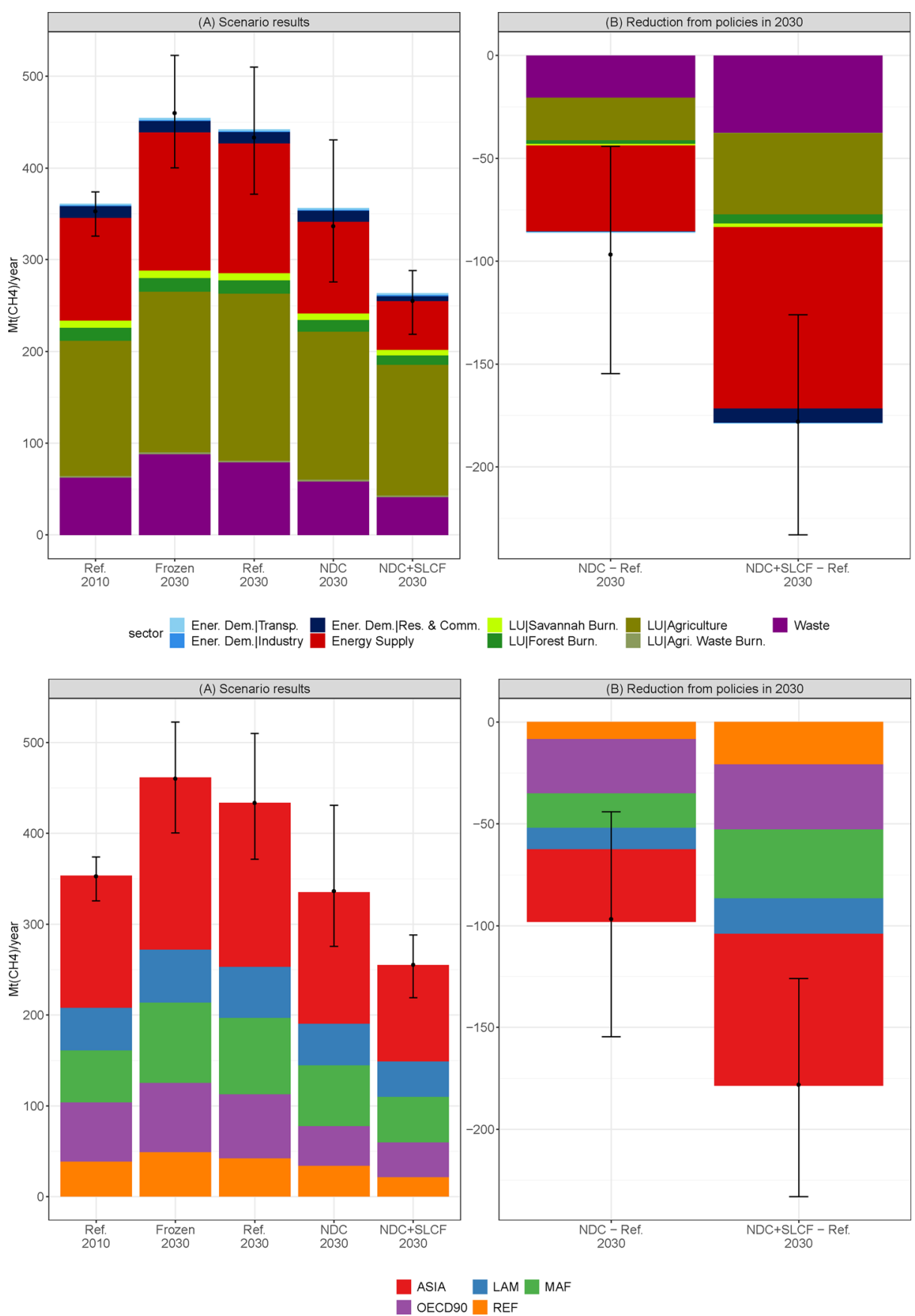

Fig. $2 \mathrm{CH}_{4}$ emissions in the NDC scenarios. Left panels: emissions in 2030, with 2010 as a benchmark. Right panels: reduction from SLCF policies compared with the reference scenario. Upper panels: sectoral emissions (energy demand $=$ blue, energy supply $=$ red, land-use $=$ green, waste $=$ purple). Lower panels: regional emissions (bars show model means, whiskers indicate minimum, maximum, and median values) (regions: see Supplement S3 for overview, LAM = Latin America, MAF = Middle East and Africa, REF = Reforming economies of former Soviet Union) 
and NDC extrapolated scenarios, as these follow the same trajectory until 2030. The bars show the model means per sector and region and model range in total emissions (individual model results are provided in Supplement S4).

In the NDC scenario, $\mathrm{CH}_{4}$ emission reductions are found in all sectors (44 to $155-\mathrm{Mt}^{\mathrm{CH}_{4}}$ or an average $20 \%$ reduction, compared with the reference case) and are the largest in fossil fuel production both in absolute and relative terms (29\% of the energy supply emissions on average). With SLCF policy, additional reductions are realized in all sectors (125 to 235-Mt $\mathrm{CH}_{4}$ or an average $41 \%$ in total), leading to lower emissions in 2030 compared with 2010 for all models. Also, in the NDC + SLCF case, emission reductions are mainly realized in the fossil fuel sectors (59\% of the energy supply emissions on average, compared with reference). Partly, this is achieved by direct $\mathrm{CH}_{4}$ measures, and partly, it is an indirect impact of $\mathrm{CO}_{2}$ policy. Generally, the models estimate slightly larger reductions from the former activity; however, there is a large model spread in the indirect effects of $\mathrm{CO}_{2}$ mitigation on $\mathrm{CH}_{4}$ emissions. ${ }^{1}$ Roughly half the emissions come from land-use sources in the historical and reference case, whereas the relative share of land-use emissions increases as climate policy intensifies, due to the larger challenges surrounding land-use mitigation (predominantly enteric fermentation in ruminants, which is approximately two thirds of the land-use $\mathrm{CH}_{4}$ ). Note that agricultural emission reductions in the $2 \mathrm{degC}$ and SLCF scenarios largely come from end-ofpipe measures (lowering emission factors, rather than activities), and a large global decrease in ruminant meat and dairy demand could further bring down emissions (see Supplement S5 for further discussion).

About $40 \%$ of the emissions originate from Asia (Fig. 2). In the NDC scenario, however, emission reductions in Asia are expected to be relatively modest. Due to the assumption of global methane actions in NDC + SLCF, this increases significantly, and reductions are more evenly distributed over the world regions. In total, the models project an average reduction of $95 \mathrm{Mt} \mathrm{CH}_{4}$ year (or 26\%) from additional SLCF policy in 2030.

\subsubsection{Black carbon emissions}

Figure 3 gives an overview of the sectoral and regional $\mathrm{BC}$ emissions, with a similar setup as Fig. 2. In the NDC scenario, $\mathrm{BC}$ emissions are only slightly reduced, due to $\mathrm{CO}_{2}$ reduction measures (3\% on average, compared with reference). In the NDC + SLCF case, BC emissions are reduced considerably (1.1- to 4.3-Mt BC or an average $34 \%$ in total). The largest mitigation options are the reduction of emissions from coal and traditional biomass use in the residential sector (on average, $70 \%$ reduction). Further emission reductions in transport sector are more limited, since even in several developing countries relatively stringent emission standards are assumed in the reference case, especially for road vehicles.

$\mathrm{BC}$ emissions in the NDC case are highest in Asia and Africa on average 49 and $32 \%$ of the total emissions in 2010 and 48 and $39 \%$ of the total emissions in the reference case in 2030. However, $95 \%$ of the reduction potential in the NDC + SLCF case in 2030 is also found in these regions (65\% in Asia, 30\% in Africa). In total, the models project an average reduction of 2.1-Mt BC (or 32\%) from additional SLCF policy in 2030. In Asia, the relative reduction in $\mathrm{NDC}+\mathrm{SLCF}$ is found to be higher than the global average: $42 \%$.

\footnotetext{
${ }^{1}$ This is explored further in a forthcoming EMF30 study on methane reduction potentials (Harmsen et al., under review). The $\mathrm{CO}_{2}$ indirect reductions are roughly half as large as reductions from direct $\mathrm{CH}_{4}$ mitigation and are projected to be 9 to $27 \%$ compared with reference.
} 

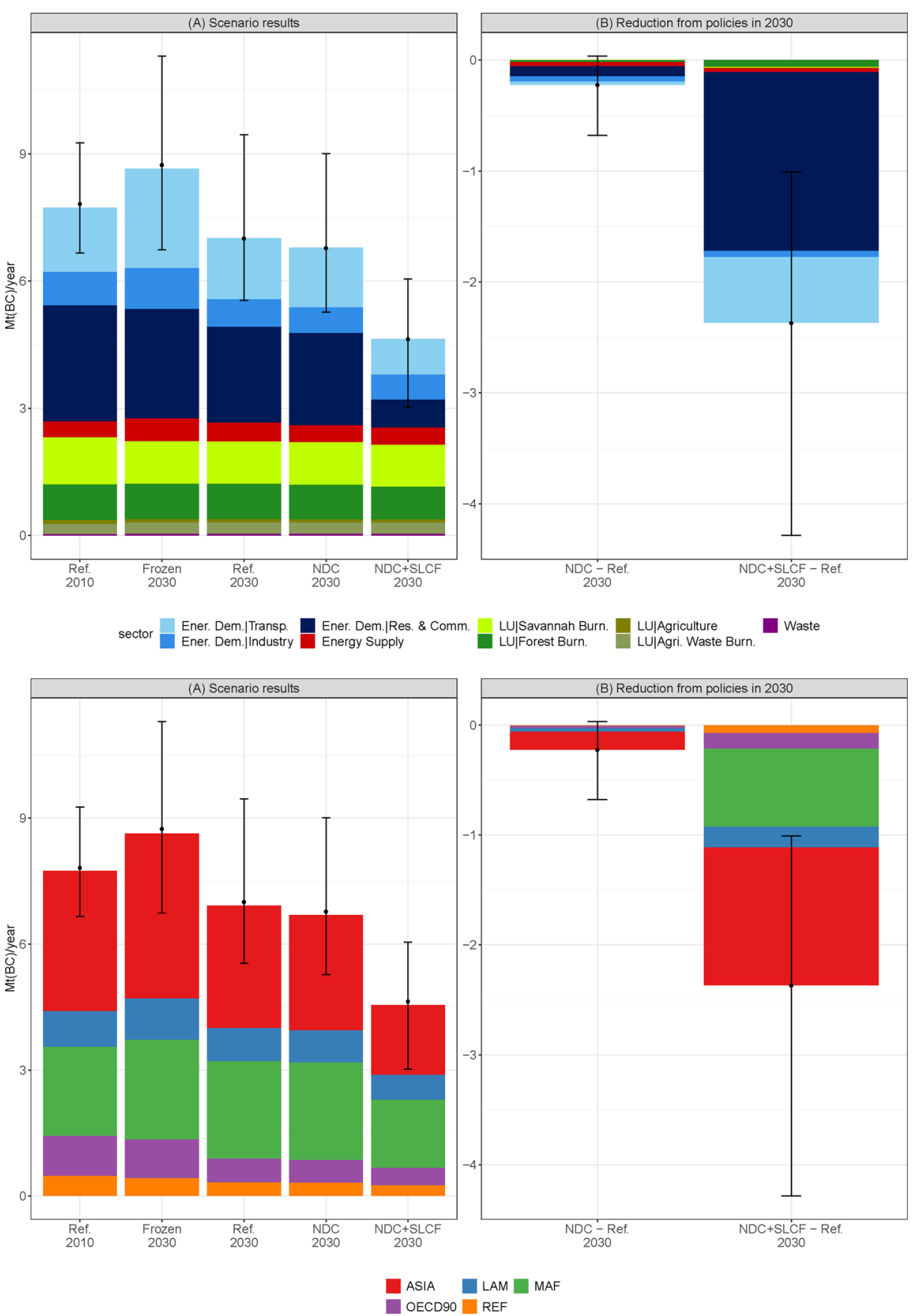

Fig. 3 BC emission reductions NDC scenarios. Left panels: emissions in 2030, with 2010 as a benchmark. Right panels: reduction from SLCF policies compared with the reference scenario. Upper panels: sectoral emissions (energy demand $=$ blue, energy supply $=$ red, land-use $=$ green, waste $=$ purple). Lower panels: regional emissions (bars show model means, whiskers indicate minimum, maximum, and median values) (regions: see Supplement S3 for overview, LAM = Latin America, MAF = Middle East and Africa, REF = Reforming economies of former Soviet Union) 
The selected $\mathrm{BC}$ reduction measures also reduce $\mathrm{OC}$ emissions, however to a lesser extent in relative terms: $22 \%$ or $4.7 \mathrm{Mt}$ of total OC emissions in the NDC + SLCF (not shown).

\subsubsection{Hydrofluorocarbon emissions}

HFC emission reduction potentials and measures vary considerably across the different models. Partly, this is because of the historically small contribution of HFCs to global warming; partly, this has resulted from uncertainty about the inclusion of HFCs in the Montreal protocol. Supplement S6 shows the HFC emission pathways for the models that ran the additional HFC reduction scenario (NDC $+2 \mathrm{DC}+\mathrm{SLCF}+\mathrm{HFC})$. All models indicate that without strong climate policy, HFC emissions would either remain constant or steadily increase. Note that the model projections exclude the recently pledged reductions under the Kigali Amendment to the Montreal protocol (UNEP 2016b), and mitigation of HFC is dependent on the carbon price development. The emissions in the NDC case are projected to be at or slightly below the no policy reference level (by AIM and REMIND) or stabilize around 800- to 1000-kt HFC134a equivalent emissions per year (by IMAGE and MESSAGE). For the 2 degree scenarios, four models project a large short-term potential for HFC reduction compared with the baseline emissions in 2030 around $88-89 \%$ for AIM/CGE, IMAGE, REMIND, and POLES in line with Höglund-Isaksson et al. (2017), who estimated this at $80 \%$. Early, ambitious HFC reduction could lead to substantially lower emissions (in 2030: $80-88 \%$ lower than NDC $+2 \mathrm{degC}$ for the four models). This reduction estimate is significantly higher (in 2030: 30-35\%) than what can be expected from the Kigali Amendment (in 2030: 54-58\% reduction (Höglund-Isaksson et al. 2017; PBL 2015). As such, considering very deep HFC reductions as a SLCF measure additional to the Kigali Amendment and the NDCs seems legitimate.

DNE21+ projects the least HFC mitigation in the HFC reduction scenario. This follows from the use of US-EPA marginal abatement cost curves (US-EPA 2013) not only with more conservative estimates of the reduction potential but also with most reductions occurring at lower carbon prices, so most reductions occur already in the NDC scenario without additional HFC policy.

The HFC emission reductions projected by MESSAGE also differ less across scenarios. MESSAGE includes mitigation options, such as refrigerant recovery, but does not represent substitution by non-GHG gases. With a maximum reduction in 2030 of just below $600-\mathrm{kt}$ HFC134a eq., the strong HFC mitigation case in MESSAGE results in higher residual HFC emissions than in all other models, but can be considered comparable with the ambition in the Kigali Amendment.

\subsection{Climate impacts}

Figure 4 shows the GMT and GMT rate of change for the reference and NDC scenarios. In both sets, a comparison is made between the scenarios with and without additional SLCF policy. Table 2 provides detailed outcomes for all models.

In the short-term (the year 2040), when the GMT reducing effect of SLCF policy is the largest, models project a potential GMT reduction of $0.03-0.15{ }^{\circ} \mathrm{C}$ in $\mathrm{NDC}+\mathrm{SLCF}$ and $\mathrm{NDC}+2 \mathrm{degC}+\mathrm{SLCF}$ (or 2 to $9 \%$ of total GMT change, which is 6 to $30 \%$ of the GMT increase between now and 2040, when considering the NDC scenario as a benchmark). This effect is projected to be slightly larger in the NDC than in the NDC $+2 \operatorname{degC}$ scenario, due to a 

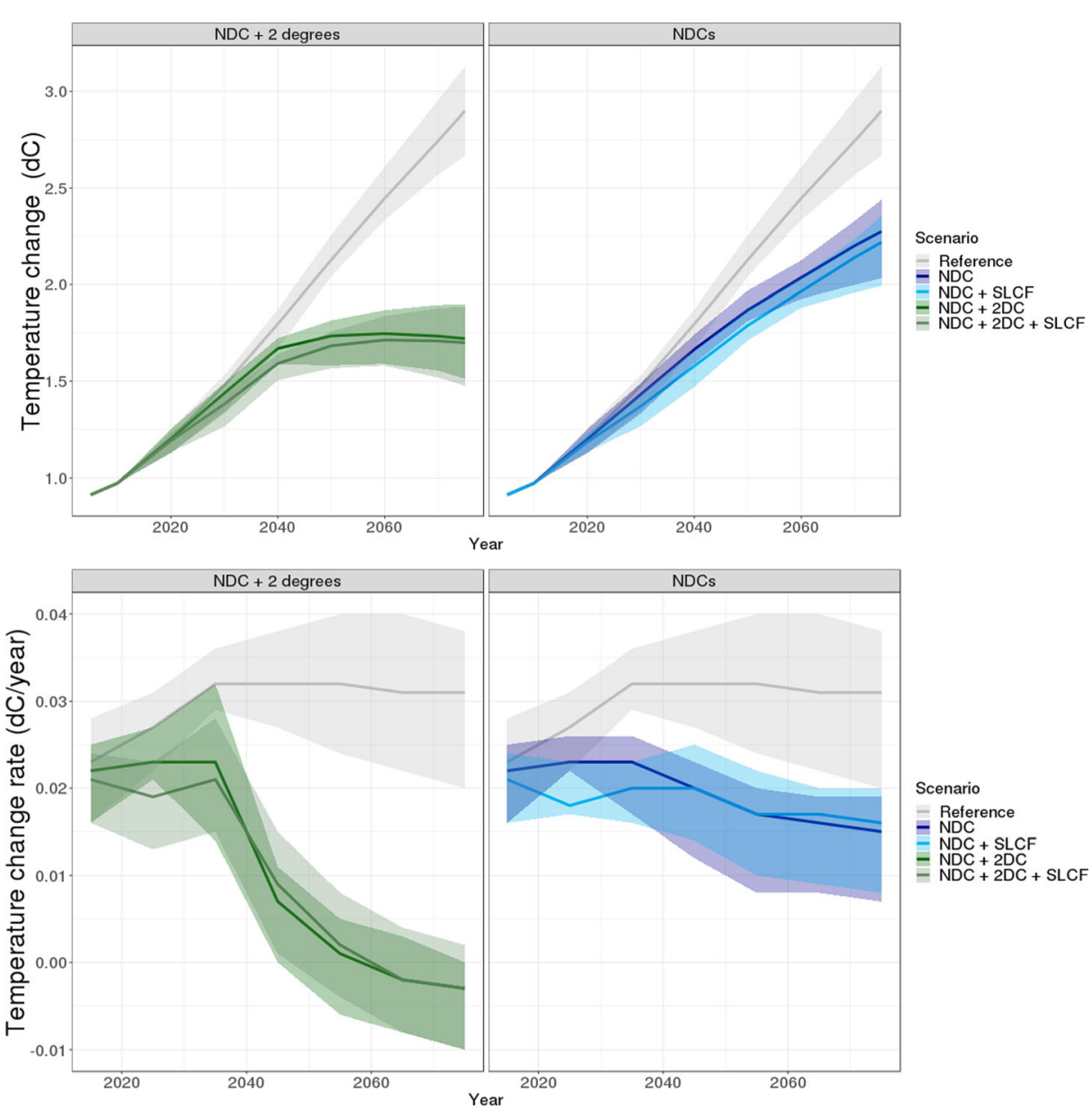

Fig. 4 Global mean temperature (GMT) and GMT rate of change in the NDC scenarios. Series shown until 2075. Upper panels: GMT difference compared with pre-industrial levels. Lower panels: GMT rate of change. General info: right $=\mathrm{NDC}$ scenarios, left $=\mathrm{NDC}+2 \mathrm{degC}$ Celsius scenarios. The no-climate policy case is shown as a reference. Lines represent the model ensemble mean, areas the ensemble range. Note: small GMT differences are projected before 2015 as carbon prices, and emission reductions are in some cases interpolated to several preceding years

lower SLCF reduction potential in the latter 2 degree case, where the SLCF and no-SLCF case converge stronger after 2030.

In an NDC $+2 d e g C+$ SLCF scenario, SLCF policy is found to have a relatively small effect on reducing the maximum temperature before 2100 (1 to $3 \%$ of total GMT change or 0.02 to $0.08^{\circ} \mathrm{C}$ ), confirming earlier work (Bowerman et al. 2013). The main reason also here is that later in the century, SLCF reductions are almost the same as in the NDC $+2 \mathrm{degC}$ case, because models exhaust their assumed mitigation options (under a sufficiently high carbon equivalent price), with maximized direct $\mathrm{CH}_{4}$ reduction measures and indirect $\mathrm{CH}_{4}$ reductions due to $\mathrm{CO}_{2}$ mitigation. This is also shown in Fig. 4, where the two 2 degree scenarios converge near 2060. Table 2 shows that most models project that additional SLCF policy will cause the GMT peak year to occur a few years later in time ( 3 on average, ranging from -5 to 8 years). 
Table 2 Impact of additional SLCF policies on GMT

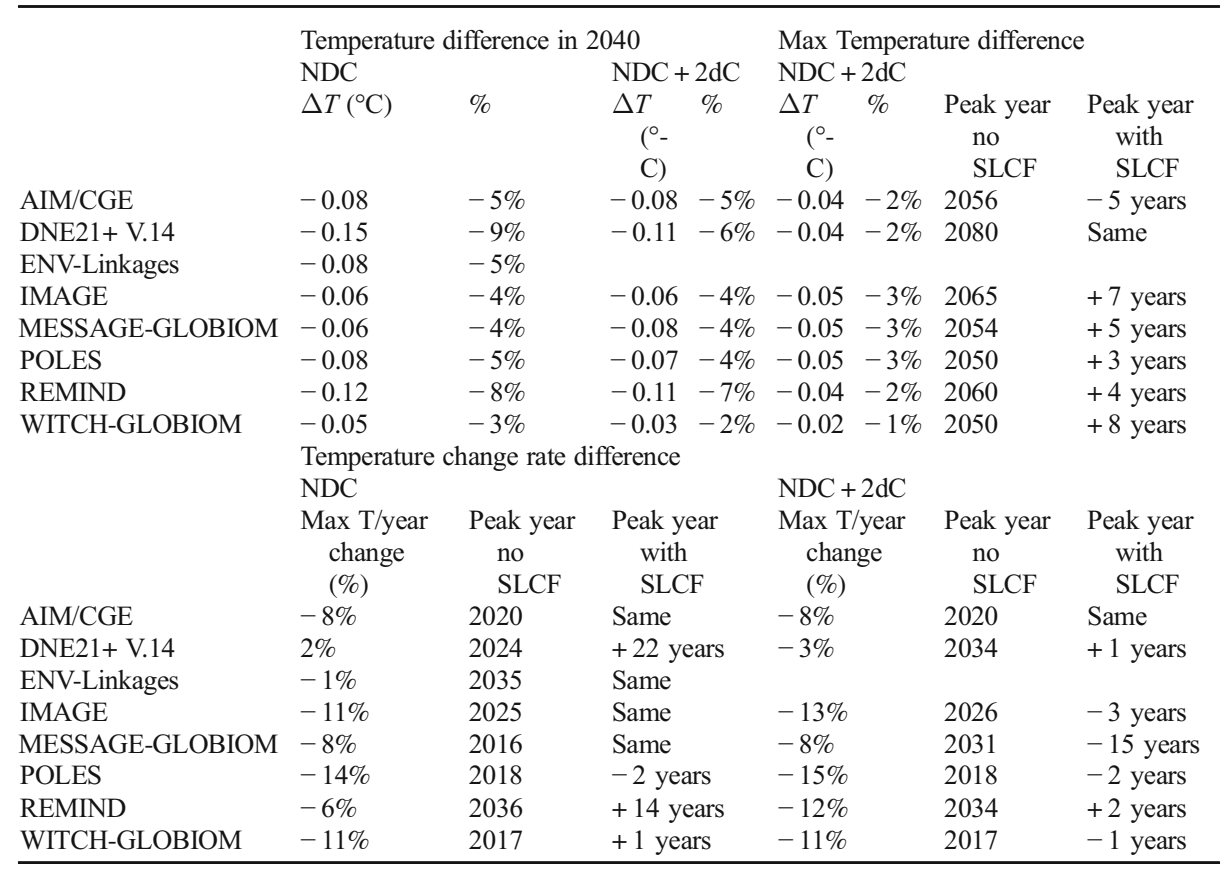

Upper panel: difference in GMT between scenarios with and without SLCF policy (in ${ }^{\circ} \mathrm{C}$ and \%). Provided for both the NDC extrapolated and NDC + 2degC case, in 2040 and in the peak temperature year (the latter only in the NDC + 2degC case, where GMT peaks before 2100). The last two columns give the timing of the GMT peak, with and without SLCF policy. Lower panel: difference in maximum GMT change rate between scenarios with and without SLCF policy (in \%). Provided for both the NDC extrapolated and NDC $+2 \mathrm{degC}$ case. The peak year columns give the timing of the GMT change rate peak, with and without SLCF policy

While the effect on lowering peak GMT is limited, the selected SLCF measures can contribute to lowering the maximum GMT change rate in both the continued NDC scenario ( 2 to $-15 \%$ change in the seven IAMs) as in the $\mathrm{NDC}+2 \operatorname{deg} \mathrm{C}$ scenario $(-3$ to $-15 \%$ change)(see lower panel Table 2). In the NDC $+2 \operatorname{deg} C$ case for all models, this peak in GMT change rate occurs before 2040 and in most cases between 2020 and 2030. Most models do not project a change in the timing of the maximum GMT rate of change due to additional SLCF reductions. If the peak value without SLCF policy is expected in the next decade, this is generally not expected to change when SLCF policy is employed. Notable exceptions are projections from DNE21+ and REMIND of the NDC case where SLCF policy shifts the maximum rate of change to mid-century. In the $\mathrm{NDC}+2 \mathrm{degC}$ case, where the highest GMT rate of change generally is projected to occur in this decade, no major shifts in peak rate of change have been projected.

An assessment of the RF levels of individual SLCF groups, $\mathrm{CH}_{4}, \mathrm{BC} / \mathrm{OC}, \mathrm{HFCs}$, and tropospheric ozone $\left(\mathrm{O}_{3}\right)$, shows that all three SLCF groups can contribute to lowering GMT $\left(\mathrm{O}_{3}\right.$ is indirectly decreased by lowering $\mathrm{CH}_{4}$ and nitrogen oxide $\left(\mathrm{NO}_{\mathrm{x}}\right)$, which is co-emitted with BC and OC). In Fig. 5, the decrease in RF resulting from SLCF measures in the NDC + $2 \mathrm{degC}$ scenarios is shown (model specific results are provided in Supplement S7). The figure shows the average RF difference between "NDC $+2 \operatorname{degC}$ " and "NDC $+2 \mathrm{degC}+\mathrm{SLCF}+$ HFCs" and is thus only based on results from the seven models that submitted the latter 


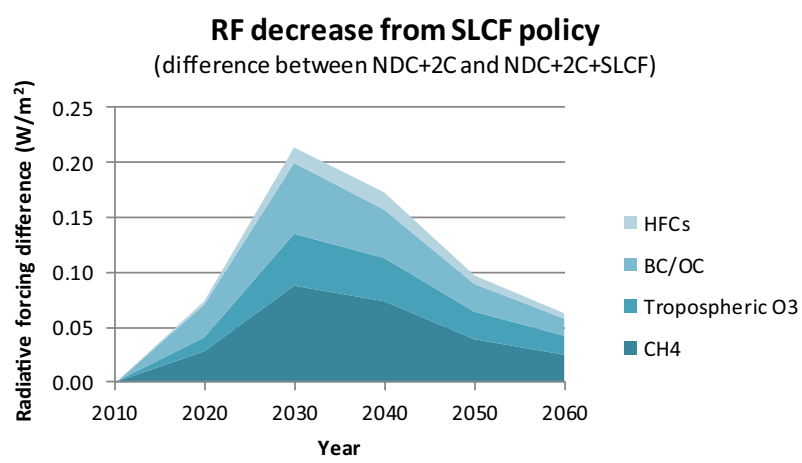

Fig. 5 Decrease in radiative forcing from SLCF policy in the NDC $+2 \mathrm{degC}$ scenario. The values represent the average of all model outcomes for the models that submitted "NDC $+2 \operatorname{degC}+\mathrm{SLCF}+\mathrm{HFCs}$ " (see Supplement $\mathrm{S} 7$ for model specific results). The $\mathrm{BC} / \mathrm{OC}$ forcing difference includes direct and indirect (cloud forming) effects from all aerosols affected by BC/OC policy. In 2030, when maximum radiative forcing reduction occurs, the relative impact of the SLCFs is methane: $41 \%$, ozone: $27 \%$, BC/OC: $26 \%$, and HFCs: $6 \%$

scenario. The relative contribution of forcers is similar in the NDC extrapolated case (not shown). The largest RF reducing effect occurs in 2030. In that year, the relative impact of additional methane reduction is $41 \%$, of $\mathrm{BC} / \mathrm{OC}$ reduction $26 \%$ and of $\mathrm{HFC}$ reduction $6 \%$. A large share of the decrease in $\mathrm{RF}$ can be accounted to a reduction in $\mathrm{O}_{3}(27 \%)$, which is mainly caused by the reduction of the $\mathrm{O}_{3}$ precursors $\mathrm{NO}_{\mathrm{x}}$ and $\mathrm{CH}_{4}$. Depending on the abundance of other species, $\mathrm{NO}_{\mathrm{x}}$ reduction is generally dominant in the reduction of $\mathrm{O}_{3}$. For instance, reducing projected $\mathrm{NO}_{\mathrm{x}}$ levels in 2100 to the 2000 value is projected to more than halve the ozone abundance (Ehhalt et al. 2001).

All models project a substantial forcing reduction that can be attributed to $\mathrm{CH}_{4}$. Considering that still a substantial part of the $\mathrm{O}_{3}$ forcing reduction is indirectly caused by $\mathrm{CH}_{4}$ reduction, more than half of the total forcing reduction comes from $\mathrm{CH}_{4}$ mitigation.

With the exception of DNE21+, the models agree on the smaller effect of BC measures. While the direct $\mathrm{BC}$ forcing decrease can be considerable (roughly twice as large as $\mathrm{BC} / \mathrm{OC}$ forcing decrease in Fig. 3), several effects reduce the net impact. Partly, it results from the reduction of OC. Secondly, aerosol emission reductions (i.e. BC, OC, nitrate, and to al smaller extent sulphate, all lowered by BC measures) are projected to decrease the cloud indirect (negative) forcing effect, thus increase forcing. For example, in MESSAGE, the effect of BC/ $\mathrm{OC}$ is relatively small. While $\mathrm{BC}$ reduction in the SLCF case is considerable, OC reduction is very large as well. Due to large aerosol emission reductions, the cloud indirect aerosol forcing is considerably increased, adding up to a small net forcing decrease. In DNE21+, the opposite is happening. Here, there is also a very large and sustained difference in $\mathrm{BC}$ emissions between $\mathrm{NDC}+2 \mathrm{DC}$ and NDC + $2 \mathrm{DC}+\mathrm{SLCF}$. However, OC and other aerosols are mitigated much less, leading to a much smaller change in $\mathrm{OC}$ direct and aerosol indirect effect.

The absolute impact of HFC reductions appears to be limited; however, the model differences in terms of emission reductions are relatively large. Models that assume a reduction potential of more than $80 \%$ of the HFCs (AIM, IMAGE, POLES, WITCH-GLOBIOM) project a RF decrease of roughly $0.03 \mathrm{~W} / \mathrm{m}^{2}$, which is roughly $15 \%$ of the total decrease. Considering that this percentage would be achieved when maximizing HFC reductions (in 2030, far beyond the Kigali amendment of the Montreal protocol), HFC abatement measures can potentially provide only a much smaller additional contribution to reducing GMT than more important $\mathrm{CH}_{4}$ and $\mathrm{BC}$ measures. 


\section{Discussion}

\subsection{Earlier work and the role of SLCF policy}

This study can be considered consistent with recent work that has identified limitations regarding the potential temperature reduction resulting from additional SLCF policy (Rogelj et al. 2014; Smith and Mizrahi 2013; Stohl et al. 2015). Earlier found key limiting factors that also played a part in this study are as follows: (1) a more limited mitigation potential, as substantial SLCF reductions are already assumed to occur in the reference case and (2) BC reduction measures lead to a reduction of global cooling aerosols, such as $\mathrm{SO}_{2}$ and $\mathrm{OC}$, which offset the effect of $\mathrm{BC}$ mitigation.

There are several reasons for the first point (limited mitigation potential). Climate policy, in the NDCs but especially also after 2030 in a $2 \operatorname{degC}$ case, is assumed to lead to direct SLCF GHG mitigation under a $\mathrm{CO}_{2}$ eq. price. This is reinforced by the " $\mathrm{CO}_{2}$ indirect effect"; there are co-mitigated $\mathrm{CH}_{4}$ emissions from $\mathrm{CO}_{2}$ reduction that should be expected from the NDCs (via the reduction of fossil fuels and deforestation), which lower the reduction potential of additional $\mathrm{CH}_{4}$ mitigation. Lastly, $\mathrm{BC}$ emissions are assumed to decrease under air pollution policy alone, lowering the potential for further reduction.

Note that, although it is concluded here that the potential climate benefit of additional, more ambitious SLCF policy is relatively modest, we do not conclude that SLCF mitigation is unimportant for reaching long-term targets. It very much is. That importance is for instance illustrated by Collins et al. (2018) and Mengis et al. (2018), who show that differences in either the level of (notably $\mathrm{CH}_{4}$ ) mitigation or forcing have large implications for the ease of reaching ambitious $(1.5 \mathrm{dC})$ targets and the level of required $\mathrm{CO}_{2}$ mitigation. However, this would equally be the case for both the $2 \mathrm{degC}$ and the $2 \mathrm{degC}+\mathrm{SLCF}$ scenario, as towards the second half of the century both scenarios are assumed to converge in terms of SLCF mitigation.

Following earlier studies (Shindell et al. 2012; Shoemaker et al. 2013; Smith and Mizrahi 2013; UNEP and WMO 2011), we acknowledge and emphasize that SLCF policy should only function as a complement to and not a substitution of long-lived climate forcer (LLCF) policy, to effectively reach long-term climate goals. A stronger focus on $\mathrm{CO}_{2}$ now would actually mean that long-term climate goals are easier to reach and should not be discouraged. ${ }^{2}$ However, Shoemaker et al. (2013) state that there is legitimate concern that countries could introduce SLCF policy to shield them from international pressure to reduce $\mathrm{CO}_{2}$ emissions. If such behaviour would occur, SLCF climate policy could actually be counter effective. Conversely, Victor et al. (2015) argue that the opposite is more likely; early successes with SLCF policies would function as a confidence builder for governments, ultimately leading to increased efforts to reduce $\mathrm{CO}_{2}$.

Note further that our focus in this study has solely been on examining the climate effects of SLCF policy. We exclude the analysis of the co-benefit of improved air quality (for human health and vegetation), which is arguably an equally important reason to reduce SLCFs.

\footnotetext{
$\overline{2}$ The sensitivity analysis in supplement $\mathrm{S} 9$ also indicates that "more $\mathrm{CO}_{2}$ focused NDCs" would lead to a stronger temperature reduction in the long term
} 


\subsection{Uncertainties in SLCF climate response}

Although the MAGICC6 model, used for this study's climate analysis, represents relevant global climate behaviour such that it fits well within the uncertainty range of complex climate models, the uncertainty in RF is very large, particularly for aerosols. In 2010, aerosol forcing is estimated at $-1.08 \pm 0.43 \mathrm{~W} / \mathrm{m}^{2}$ (ACCMIP 2013). The MAGICC6 median value used in our study falls in the middle of this range $\left(-1.09 \mathrm{~W} / \mathrm{m}^{2}\right)$. However, the effect of $\mathrm{BC}$ measures, which affect a whole range of aerosol species, can potentially have been underestimated or overestimated. Recent model and field studies suggest the latter and consistently indicate that direct BC RF (Samset et al. 2014) and effective RF (proportional to GMT change) (Baker et al. 2015; Stjern et al. 2017) are likely on the low side of their uncertainty ranges.

The climatic effect of additional BC measures in the long-term is also expected to remain limited due to a net global decrease in pollutant emissions in all scenarios, by assuming further strengthening of air quality legislation across the developing world (Rao et al. 2017; Rogelj et al. 2014; Smith and Bond 2014).

The climate impacts from $\mathrm{CH}_{4}$ are considered significantly more robust than those of aerosols. However, recent studies might call for a revision in the climatic role of $\mathrm{CH}_{4}$. Etminan et al. (2016) suggested that the $\mathrm{CH}_{4}$ present day $\mathrm{RF}$ has been historically underestimated and should be increased by $25 \%\left(0.48\right.$ to $\left.0.61 \mathrm{~W} / \mathrm{m}^{2}\right)$. In addition, Modak et al. (2018) estimated a smaller efficacy (effective impact of RF on GMT) for $\mathrm{CH}_{4}$ (17\% lower than $\mathrm{CO}_{2}$ and earlier assumed). These factors may partially compensate for each other in terms of impact on GMT.

In order to understand the range in potential climate impacts of SLCF mitigation measures, Supplement S10 provides an uncertainty analysis of the two 2 degree scenarios (NDC $+2 \mathrm{degC}$ and $\mathrm{NDC}+2 \operatorname{deg} \mathrm{C}+\mathrm{SLCF}$ ), as this also allows for an assessment of the peak GMT lowering potential of the SLCF measures. In a Monte Carlo analysis, all IAM model versions of the two 2 degree scenarios have been run with a probabilistic version of MAGICC6 with 600 cases per scenario that represent alternate configurations of 82 climate model parameters (climate sensitivity, ocean heat diffusion rate, and RF strength of aerosol and GHG species) resulting in a range of possible GMT changes (see Fig. S10.1).

It can be concluded that the maximum GMT reducing potential of the SLCF measures is likely not much higher than the highest model projections in the original MAGICC runs (as in Fig. 4). Although for each individual scenario, the uncertainty in GMT change is very large (with a range in 2040 larger than $0.6^{\circ} \mathrm{C}$ ), the GMT difference between the two 2 degree scenarios (i.e. the effect of SLCF measures) will likely not exceed $0.13{ }^{\circ} \mathrm{C}$ (i.e. the maximum value found in 2040), when implementing the mitigation measures considered in this study. At the lower end of the range, the GMT difference between the two 2 degree scenarios is almost negligible $\left(0.002{ }^{\circ} \mathrm{C}\right.$ in 2040$)$, implying no significant effect from additional SLCF measures in this extreme case. Uncertainty in the peak GMT lowering potential of the SLCF measures is smaller and ranges between 0 and $0.055{ }^{\circ} \mathrm{C}$ (largely around the year 2060).

\subsection{A potential larger impact from additional SLCF reduction?}

For a background analysis of this section, please find "assessment of NDCs and national climate plans" in Supplement S8. In it, existing and intended national non- $\mathrm{CO}_{2}$ policies have been described and compared with projected non- $\mathrm{CO}_{2}$ policy in this study's scenario analysis.

The IAMs that have taken part in the scenario assessment employ mitigation options based on a least-cost approach based on global warming potential (GWP) weighting (see 
Supplement, Table S1.2). For most models, this makes it relatively attractive to mitigate methane at low carbon prices. While it is possible that this also occurs in reality, it is also likely that countries largely stick to the policies in their national plans, which, as is shown in the analysis in the SI, often do not explicitly mention $\mathrm{CH}_{4}$ measures and have a stronger focus on energy and $\mathrm{CO}_{2}$. If such a strong $\mathrm{CO}_{2}$ focus would remain, the additional effect of SLCF policy would be larger. To assess this potentially larger impact, a sensitivity analysis was performed with the IMAGE IAM and MAGICC6.3 (Supplement S9). For the NDC + 2degC case, this is most relevant, since peak GMT can potentially be lowered. It is shown that in an extreme case (with no non- $\mathrm{CO}_{2}$ policy assumed when it is not explicitly embedded in national policies), the GMT reducing potential as well as the lowering of the peak GMT could theoretically be doubled. However, it is considered unlikely that no non- $\mathrm{CO}_{2}$ policies whatsoever would be introduced in the reference NDC case, so a much smaller (but uncertain) increase in the GMT decreasing potential of SLCFs would be more likely. The analysis also shows that the projected minimum GMT under SLCF policy is relatively robust, with no potential error larger than $0.008{ }^{\circ} \mathrm{C}$. Only if the models currently underestimate the short-term mitigation potential of SLCFs (e.g. a larger potential for lowering emissions from meat and dairy), the minimum GMT could be lower.

\section{Conclusions}

This study examines the climate impact of intensified SLCF policy, using the existing global climate policy regime, the NDCs, as a starting point. While most countries have formulated their NDCs in terms of reducing all GHGs, it is found that national policies generally do not explicitly target non- $\mathrm{CO}_{2}$ emissions. However, due to the expected cost-effectiveness of many non- $\mathrm{CO}_{2}$ reduction measures, the models used in this study often favour SLCF (i.e. methane) measures in policy strategies. Based on the model assessment, this study finds that:

- $\quad$ The proposed SLCF measures, additional to estimated NDCs implementation, can contribute to reducing GMT in the short-term (the year 2040): by 0.03 to $0.15^{\circ} \mathrm{C}$. This effect is projected to be slightly larger in an NDC case than in an NDC $+2 \operatorname{degC}$ scenario. The GMT reducing effect of additional SLCF policies could be higher (up to twice as high, but likely much less) if countries primarily use $\mathrm{CO}_{2}$ mitigation to give substance to their NDCs.

- The measures are found to have only a small effect on reducing the maximum temperature before 2100 (roughly one third of the short-term effect); mostly because in the second half of the century under assumed ambitious climate policy in a $2 \operatorname{degC}$ case, the projected SLCF mitigation is maximized, due to high $\mathrm{CO}_{2}$-equivalent pricing, either directly, or indirectly resulting from decarbonization changes in the energy system.

- Maximizing SLCF mitigation can reduce the maximum temperature rate of change in the short term, by -2 to $15 \%$. All models show that the short-term reduction in temperature rate of change is particularly relevant in an NDC $+2 \mathrm{degC}$ case. In a continued NDC case, the effect is less certain, and some models project a delay, but not a decrease in the temperature rate of change.

- All three SLCF groups can potentially contribute to these effects, although methane measures have the largest impact $(>50 \%$ of the total). The impact of additional HFC reduction is likely to be limited $(<10 \%)$, especially if the recent inclusion of HFC reduction under the Montreal protocol (i.e. Kigali Amendment) will prove successful. 
- $\quad$ The temperature reducing potential from SLCFs as found in this study can be considered relatively limited compared with that of $\mathrm{CO}_{2}$ mitigation, which is consistent with recent studies. Following earlier studies, we also emphasize that SLCF policy should only function as a complement to and not a substitution of LLCF policy, to effectively reach long-term climate goals. The conclusions from this study only hold if such additionality can be assured.

Funding information The research leading to these results has received funding from the Climate Works Foundation (IIA/17/1303). We would like to thank them and our colleagues who have directly or indirectly supported the work that has been done within the EMF30 consortium.

Open Access This article is distributed under the terms of the Creative Commons Attribution 4.0 International License (http://creativecommons.org/licenses/by/4.0/), which permits unrestricted use, distribution, and reproduction in any medium, provided you give appropriate credit to the original author(s) and the source, provide a link to the Creative Commons license, and indicate if changes were made.

\section{References}

ACCMIP (2013) Atmospheric Chemistry and Climate Model Intercomparison Project. http://www.giss.nasa. gov/projects/accmip/publications.html. Accessed 24 Apr 2018

Baker LH et al (2015) Climate responses to anthropogenic emissions of short-lived climate pollutants. Atmos Chem Phys 15:8201-8216

Bond TC et al (2013) Bounding the role of black carbon in the climate system: a scientific assessment. J Geophys Res Atmos 118:5380-5552

Bowerman NHA et al (2013) The role of short-lived climate pollutants in meeting temperature goals. Nat Clim Chang 3:1021-1024

Collins WJ et al (2018) Increased importance of methane reduction for a 1.5 degree target. Environ Res Lett 13:054003

Den Elzen M et al (2016) Contribution of the G20 economies to the global impact of the Paris Agreement climate proposals. Clim Chang 137:655-656

Ehhalt D et al (2001) Atmospheric chemistry and greenhouse gases. In: Climate change 2001: the scientific basis. Cambridge University Press, Cambridge, p 892

EMF (2017) EMF 30: short lived climate forcers/air quality

Etminan $\mathrm{M}$ et al (2016) Radiative forcing of carbon dioxide, methane, and nitrous oxide: a significant revision of the methane radiative forcing. Geophys Res Lett 43

Fawcett AA et al (2016) Can Paris pledges avert severe climate change? Reducing risks of severe outcomes and improving chances of limiting warming to $2{ }^{\circ} \mathrm{C}$. Science 350

Harmsen $\mathrm{M}$ et al (2015) How well do integrated assessment models represent non- $\mathrm{CO}_{2}$ radiative forcing? Clim Chang 133:565-582

Höglund-Isaksson L et al (2017) Cost estimates of the Kigali Amendment to phase-down hydrofluorocarbons. Environ Sci Pol 75:138-147

Kriegler E et al (2014) Making or breaking climate targets: the AMPERE study on staged accession scenarios for climate policy. Technol Forecast Soc Chang

Meinshausen $\mathrm{M}$ et al (2011) Emulating coupled atmosphere-ocean and carbon cycle models with a simpler model, MAGICC6: part I - model description and calibration. Atmos Chem Phys 11:1417-1456

Mengis $\mathrm{N}$ et al (2018) 1.5 degrees $\mathrm{C}$ carbon budget dependent on carbon cycle uncertainty and future non- $\mathrm{CO}_{2}$ forcing. Sci Rep 8:5831

Modak A et al (2018) Does shortwave absorption by methane influence its effectiveness? Clim Dyn

PBL (2015) Climate action outside the UNFCCC, Assessment of the impact of international cooperative initiatives on greenhouse gas emissions. PBL Policy brief. Publication number 1188

Pierrehumbert RT (2014) Short-lived climate pollution. Annu Rev Earth Planet Sci 42

Rao S et al (2016) A multi-model assessment of the co-benefits of climate mitigation for global air quality. Environ Res Lett 11 
Rao S et al (2017) Future air pollution in the shared socio-economic pathways. Glob Environ Chang

Riahi K et al (2017) The shared socioeconomic pathways and their energy, land use, and greenhouse gas emissions implications: an overview. Glob Environ Chang

Rogelj $\mathrm{J}$ et al (2014) Disentangling the effects of $\mathrm{CO}_{2}$ and short-lived climate forcer mitigation. PNAS 111: $16325-16330$

Rogelj J et al (2016) Paris Agreement climate proposals need a boost to keep warming well below $2{ }^{\circ} \mathrm{C}$. Nature 534

Samset BH et al (2014) Modelled black carbon radiative forcing and atmospheric lifetime in AeroCom phase II constrained by aircraft observations. Atmos Chem Phys 14:12465-12477

Shindell D et al (2012) Simultaneously mitigating near-term climate change and improving human health and food security. Science 335:183-189

Shindell D et al (2017) A climate policy pathway for near- and long-term benefits. Science 356:493-494

Shoemaker JK et al (2013) What role for short-lived climate pollutants in mitigation policy? Science 342:13231324

Smith SJ, Bond TC (2014) Two hundred fifty years of aerosols and climate: the end of the age of aerosols. Atmos Chem Phys 14:537-549

Smith SJ, Mizrahi A (2013) Near-term climate mitigation by short-lived forcers. PNAS 110:14202-14206

Stjern CW et al (2017) Rapid adjustments cause weak surface temperature response to increased black carbon concentrations. J Geophys Res Atmos 122:462-481

Stohl A et al (2015) Evaluating the climate and air quality impacts of short-lived pollutants. Atmos Chem Phys 15:10529-10566

Strefler J et al (2014) Economic impacts of alternative greenhouse gas emission metrics: a model-based assessment. Clim Chang 125:319-331

Tavoni $\mathrm{M}$ et al (2014) Post-2020 climate agreements in the major economies assessed in the light of global models. Nat Clim Chang 5:119-126

UNEP (2016a) The emissions gap report 2016. United Nations Environment Programme. Nations Environment Programme (UNEP), Nairobi

UNEP (2016b) The Kigali amendment to the Montreal protocol: HFC phase-down. OzonAction, UN Environment (UNEP), Economy Division

UNEP, WMO (2011) Integrated assessment of black carbon and tropospheric ozone: summary for decision makers. United Nations Environment Programme, Nairobi, Kenya

UNFCCC (2015a) Intended nationally determined contributions (INDCs). http://www4.unfecc. int/submissions/indc/Submission\%20Pages/submissions.aspx, Accessed: 29 October, 2015

UNFCCC (2015b) Paris Agreement. Decision 1/CP.17. http://unfccc.int/resource/docs/2015/cop21/eng/109r01. pdf. Accessed 12 Dec 2015, UNFCCC document FCCC/CP/2015/L.9/Rev.1

US-EPA (2013) United States Environmental Protection Agency (USEPA), global mitigation of non- $\mathrm{CO}_{2}$ greenhouse gases: 2010-2030

Vandyck T et al (2016) A global stocktake of the Paris pledges: implications for energy systems and economy. Glob Environ Chang 41:46-63

Victor D et al (2012) The climate threat we can beat. Foreign Aff 91:112-121

Victor D et al (2015) Soot and short-lived pollutants provide political opportunity. Nat Clim Chang 5:796-798

Publisher's note Springer Nature remains neutral with regard to jurisdictional claims in published maps and institutional affiliations.

\section{Affiliations}

\section{Mathijs Harmsen $^{1,2} \cdot$ Oliver Fricko $^{3}$ - Jérôme Hilaire ${ }^{4,5}$ - Detlef P. van Vuuren ${ }^{1,2}$. Laurent Drouet $^{6} \cdot$ Olivier Durand-Lasserve $^{7}$. Shinichiro Fujimori ${ }^{8,9} \cdot$ Kimon $^{2}$ Keramidas $^{10} \cdot$ Zbigniew Klimont $^{3} \cdot$ Gunnar Luderer $^{4} \cdot$ Lara Aleluia Reis $^{6} \cdot$ Keywan Riahi $^{3} \cdot$ Fuminori Sano ${ }^{11} \cdot$ Steven J. Smith ${ }^{12}$}

1 PBL Netherlands Environmental Assessment Agency, Bezuidenhoutseweg 30, 2594 AV The Hague, The Netherlands

2 Copernicus Institute for Sustainable Development, Utrecht University, Heidelberglaan 2, 3584 CS Utrecht, The Netherlands

3 International Institute for Applied Systems Analysis, Schlossplatz-1, A-2361 Laxenburg, Austria 
4 Potsdam Institute for Climate Impact Research (PIK), Member of the Leibniz Association, POBox 601203 , D-14412 Potsdam, Germany

5 Mercator Research Institute on Global Commons and Climate Change (MCC), Torgauerstrasse 12, 10829 Berlin, Germany

6 RFF-CMCC European Institute on Economics and the Environment (EIEE), Via Bergognone 34, 20144 Milan, Italy

7 OECD Environment Directorate, 2 rue André Pascal, 75116 Paris, France

8 Department Environmental Engineering, Kyoto University, 361, C1-3, Kyoto University Katsura Campus, Nishikyo-ku, Kyoto 615-8540, Japan

9 Center for Social \& Environmental Systems Research, National Institute for Environmental Studies, 16-2 Onogawa, Tsukuba, Ibaraki 305-8506, Japan

10 European Commission_-JRC, Edificio Expo, Calle Inca Garcilaso, 3, 41092 Sevilla, Spain

11 Research Institute of Innovative Technology for the Earth 9-2, Kizugawadai, Kizugawa-Shi, Kyoto 6190292, Japan

12 Joint Global Change Research Institute, Pacific Northwest National Laboratory, 5825 University Research Court, Suite 3500, College Park, MD 20740, USA 